\title{
Dialogue Coherence: A Generation Framework
}

\author{
Robbert-Jan Beun • Rogier M. van Eijk
}

Published online: 21 September 2007

(C) Springer Science+Business Media B.V. 2007

\begin{abstract}
This paper presents a framework for the generation of coherent elementary conversational sequences at the speech act level. We will embrace the notion of a cooperative dialogue game in which two players produce speech acts to transfer relevant information with respect to their commitments. Central to the approach is that participants try to achieve some sort of balanced cognitive state as a result of speech act generation and interpretation. Cognitive states of the participants change as a result of the interpretation of speech acts and these changes provoke the production of a subsequent speech act. Describing the properties and the dynamics of the mental constructs that constitute the participants' cognitive states, such as beliefs and commitments, in relation to the various dialogue contributions is an essential aspect of the game. Although simple in its basic form, the framework enables us to produce abstract conversations with some properties that agree strikingly with coherence structures found in, for instance, Conversation Analysis.
\end{abstract}

Keywords Cooperative dialogue games $\cdot$ Speech act generation $\cdot$ Cognitive state update $\cdot$ Cognitive balance $\cdot$ Commitments $\cdot$ Beliefs

\section{Introduction}

In its basic form, a dialogue can be conceived as a linear alternating sequence of symbolic elements between two participants who alternately play the role of sender and

R.-J. Beun ( $\varangle) \cdot$ R. M. van Eijk

Faculty of Science, Department of Information and Computing Sciences,

Utrecht University, P. O. Box 80089, Utrecht 3508 TB, The Netherlands

e-mail: rj@cs.uu.nl

R. M. van Eijk

e-mail: rogier@cs.uu.nl 
receiver (Hamblin 1971). The utterances in a dialogue do not form independent segments of text, but show, like words in a sentence, a coherent structure of conversational units. In both the generation and the interpretation process, dialogue participants relate the content of new contributions to the previous discourse. The construction of a coherent representation of the conversational units in a dialogue is an essential cognitive activity in the process of understanding discourse.

Coherence as a relation in discourse has been studied from many different angles. Most studies in linguistics take an analytical stance and consider, for instance, the acceptability of written texts or dialogue sequences and the type of relations that should be taken into account to build a coherent representation of these discourses (e.g., Hobbs 1979; Redeker 1990; Givón 1995; Sanders and Noordman 2000). Inspired by computational theories on natural language processing and the need for computer systems that generate cooperative dialogue contributions, coherence is nowadays also studied from the perspective of language production (see e.g., Asher and Lascarides 1998; Beun 2001; Hulstijn et al. 2005; Gatt and van Deemter 2006; Kibble and Power 2004; Piwek 2006). In these 'synthetic' theories, questions have to be answered about, for instance, the appropriateness of responses in a dialogue, the adequate realisation of referential expressions or the linear ordering of discourse units as part of a dialogue turn. In these cases, coherence is often considered as a constraint on the communicative behaviour of the participants in terms of the production of speech acts or actual realization of the linguistic form.

In line with the 'synthetic' theories, the central goal of this paper is to present a computational framework that enables us to generate coherent elementary conversational sequences at the speech act level. For that, we will embrace the notion of a cooperative dialogue game (Carlson 1985) in which two players produce speech acts or 'moves' to transfer relevant information with respect to their cognitive states. Central to the approach, and in line with (Larsson and Traum 2000), is that the cognitive states of the players change as a result of the interpretation of the speech acts (Bunt 1989) and that these changes provoke the production of a subsequent move. The game works roughly like this: A speech act is generated on the basis of preconditions formed by the cognitive state of the sender, but changes the cognitive states of both sender and addressee after it has been manifested. In the next turn the addressee adopts the sender role and, subsequently, the changed mental constructs of his or her state function as the new preconditions for the next speech act. Conversational coherence of subsequent speech acts with previous utterances is established by the cognitive state of the participants and the rules for cognitive update and dialogue behaviour. As in realistic conversational situations, it is assumed that the information relevant with respect to a particular answer can be distributed among the participants.

Describing the properties and the dynamics of the mental constructs, such as beliefs and commitments (Hamblin 1971; Singh 1999; Hulstijn 2000), in relation to the various dialogue contributions is an essential part of the work. We will show that the coherence of the speech acts is tied to local interactions contingent to the agent's particular situation and that the coherence relations can be described in a situated sense, i.e., driven by the history of the speech acts and the dynamics of the mental constructs of the participants. 
This paper is organized as follows. In Sect. 2 we will consider the notion of coherence in dialogue. In Sect. 3 we will describe the basic aspects of the generation model and the underlying theoretical principles of the dialogue game. In Sect. 4 we will describe the mental constructs used in the dialogue game and the notion of balance and in Sect. 5 the actual game is defined and in Sect. 6 an example is worked out. In Sect. 7, conclusions are given together with lines for further research.

\section{Dialogue Coherence}

Coherence relations can be described on a syntactic and a semantic level. Syntactically, most models of conversation include both a linear and a hierarchical conception of coherence relations. Linearity is established by a notion of pairing - two utterances that for some reason seem to be related to each other at the same level. In Conversation Analysis, for instance, the fundamental pairs of conversational organisation are sequences called 'adjacency pairs': a question is followed by an answer, a greeting by a counter-greeting, etcetera (e.g., Levinson 1983). From speech act theory we know the notion of 'uptake' (Austin 1962), being the dependency of a successful performance of an illocutionary act on the reaction of the addressee. Hierarchy, on the other hand, is established by embedded structures that may appear between paired units. In dialogue this can be created by so-called 'insertion sequences'-i.e., deviations from the main point that are usually expressed by the first part of an adjacency pair. Through the embedding structures of adjacency pairs, the recursive organisation of conversation becomes apparent. Similar structures can be found in, for instance, (Power 1979; Grosz and Sidner 1986; Polanyi 1988; Longacre 1996). A disadvantage of the syntactic approach is that it does not explain why particular sequences are conceived as being linear or hierarchical.

In line with, for instance, (Redeker 1990; Sanders et al. 1992; Bateman and Rondhuis 1997), we will assume that semantic coherence relations have an informational and/or an intentional nature. Informational coherence relations concern a 'believed' relation between the units that corresponds to an existing relation in the world described by the discourse (co-reference, spatio-temporal relations, causality, and the like). The informational view often refers to the participants' ontological and assertional knowledge about the discourse domain. Intentional relations do not lie in the state of affairs described, but in the assumed relations in the goals and plans of the participants (the illocutionary and perlocutionary relations, such as question-answer, offer-rejection and threat-defence).

In order to better define our intuition, we will first discuss some examples. All examples were taken from a small scale pilot experiment with 30 subjects who had to judge the degree of coherence on a scale from 1 (low coherence) to 5 (high coherence) of five short parts of Dutch discourse. The first two examples show (in)coherence at the informational level.

Ex1. I read a book. The stone is black. $(c=1.4, s d=0.6, n=30)$

where $c$ indicates the coherence score, $s d$ the standard deviation and $n$ the number of judgements. We can conclude from the low $s d$ that in this case there was much agreement between the subjects. The next example had the highest score: 
Ex2. I read a book. The writer is a wise man. $(c=4.5, s d=0.7, n=30)$

The two examples show that neither object continuation nor coherence markers in the second sentence are decisive in the subjects' judgement. Subjects judged Ex 2 as a coherent sequence, because they know that books are written by writers. In other words, since they know that there exists a relation between the objects in the first and the second sentence of Ex2, the subjects consider the two sentences as coherent utterances. In Ex 1 the relation is absent, although, with some imagination, we could think of a situation where the 'I'-person is Fred Flintstone reading a book made of black stone. This implies that in the judgement of coherence the belief (or knowledge) state of the dialogue participants should be taken into account.

Let us now take a simple dialogue example:

\section{Ex3. A: Is John in the kitchen? \\ B: What time is it?}

$(c=2, s d=1.1, n=30)$

Here, the coherence score is relatively low and there is less agreement between the subjects, but coherence is significantly higher than the coherence in Ex1 (Wilcoxon, $z=-2,20, p<0.05)$. In the interview after the experiment, subjects who gave a relatively high score to Ex 3 responded that they could imagine that $B$ knows that John is always in the kitchen at a certain time. In other words, in a dialogue two subsequent utterances were considered as being coherent if there exists an informational relation in the last speaker's mind between the interpreted content of the utterance in the previous turn and the content of the utterance of this last speaker.

The next example from Sacks (discussed in Levinson 1983):

\section{Ex4. A: I have a fourteen year old son \\ $B$ : Well, that's all right \\ A: I also have a dog \\ B: Oh I'm sorry \\ $(c=2.83, s d=1.1, n=30)$}

seems a bizarre sequence in isolation, but when embedded in a context of goals and roles $-A$ is trying to rent an apartment from landlord $B$-looks quite natural. Levinson uses the example to motivate the proposition that people have bad intuitions about the well-formedness of speech act sequences once they are taken out of the context. Although the coherence score is medium, we observe a relatively high score for $s d$ (compared to Ex1 and Ex2). In the interview, subjects who gave Ex4 a high coherence score responded that they invented some sort of background, like the tenant-landlord situation; the other subjects could not imagine a situation where the dialogue makes sense. So, in order to understand the coherence relation, we also need information about the goals of the dialogue participants. Two subsequent utterances may seem incoherent, but apparently the sequence becomes coherent when people know the common goal of the dialogue.

In a related experiment, the following example was included: 
Ex5. A: What time is it?

$B$ : I have to go to the toilet.

$(c=2.63, s d=1.2, n=30)$

The scores are in line with Ex4, but the utterances are not related in the same way. In Ex4, the landlord answers $A$ 's questions; in Ex5, $B$ indicates that he gives priority to another goal, namely releasing the pressure of his bladder to answering $A$ 's question. In other words, $B$ shifted from the common domain goal (i.e., answering the question) to a procedural goal by indicating that, for instance, the answer will be postponed. This was also the interpretation of subjects who gave the sequence a high score.

In general, dialogue coherence manifests itself as a subjective phenomenon and the judgement of coherence is a sliding scale that heavily depends on the background knowledge and goals of the dialogue participants. Also, coherence in a dialogue often cannot be established without the continuity or recurrence of semantic elements but the continuity does not have to be included in the surface structure of the utterances, as we have seen in the previous examples. So, in this paper, coherence is not considered as an intrinsic property of a text or a dialogue, but mainly as a mental phenomenon (c.f. Rickheit and Strohner 1992; Gernsbacher and Givón 1995). In the dialogues that will be generated below we will observe the elementary structural phenomenon, such as linear and hierarchical sequences based on the organization of the mental structure in terms of beliefs and goals of the participants and the rules that were used in the generation framework. Based on this framework, we will be able to show why particular sequences of discourse may be interpreted as linear and others as hierarchical. In what follows, we will describe the dialogue game and its underlying communication model.

\section{The Basic Model}

The dialogue game presented in this paper is based on a simple model employed in human-computer interaction (Hutchins 1989; Ahn et al. 1995). Underlying the model is the recognition that humans interact naturally with their environment in two ways: symbolically and physically. On the one hand, if there is an intermediary interpreter, humans can interact symbolically and use language to give commands, ask for or provide information, etcetera. On the other hand, physically, one manipulates objects, for instance, by moving or fastening them, or observes them by seeing, feeling, hearing, tasting or smelling. The essential difference between the two types of interaction is that actions of the first type need an interpreter who can bridge the gap between the symbols and their actual meaning and purpose, while actions of the second type are related in a more direct manner to human perception and action.

In parallel with the distinction symbolical versus physical, humans engaged in dialogues can perform two types of external actions: (a) communicative actions intended to cause some cognitive effect in the recipient, and (b) non-communicative actions to observe or change particular properties of the domain. Obviously, the two types of action can be considerably interrelated. In addition, we will include an action type that 


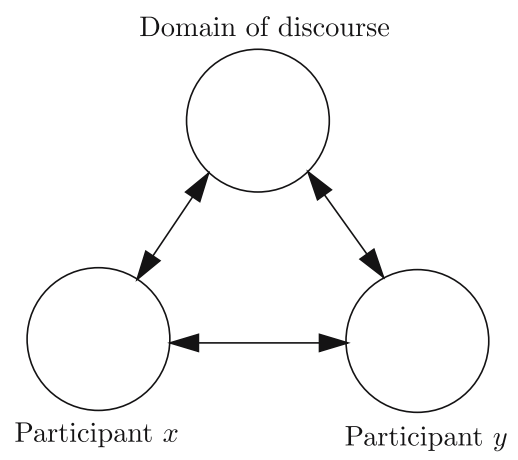

Fig. 1 The triangle metaphor

is neither communicative nor external, namely inference-i.e., the process of adjusting the cognitive states of participants solely based on their previous states. In short, the basic model includes perception, action, communication and thinking in an extremely rudimentary form.

The distinctive interaction channels are represented in the so-called triangle metaphor (Fig. 1), where the corners represent the domain of discourse (or the external world) and the two participants, and the arrows the flow of information between the participants and between the participants and the domain. A communicative act performed by participant $x$ towards participant $y$ is a flow of information from $x$ to $y$; observation of the domain is a flow of information from the domain towards the observer and an action carried out in the domain is a flow of information from the actor to the domain. In practice, the channel between the two participants may cause messages to be delayed or disturbed by, for instance, noise. Also, the channel can be duplex, where both participants can speak at a time, or half-duplex, where only one participant can speak at the time. Here, we will consider the channel between the participants and between the participants and the domain of discourse as an ideal halfduplex channel, which means that no information is delayed or lost during transfer and that information can flow only in one direction at a time.

Discourse or 'semantic' information will be divided into two categories: (a) perceivable facts (represented by single proposition letters $p, q, \ldots$ ) and (b) inference relations. Two types of inference relations will be distinguished which enable the dialogue participants to reason about their beliefs (belief inference) and their commitments (commitment inference). The commitment inference enables the participants to develop sub-commitments; for instance, $A$ wants to be outside and $A$ is inside, then A has to open the door and, consequently, 'opening the door' becomes a commitment by $A$.

The two types of inferences will be denoted as follows: belief inferences will be of the form (' $p \rightarrow q$ ',$\ldots$ ) and commitment inferences of the form (' $p \times q \rightarrow r$ ', .), connecting a simple proposition or a pair of simple propositions (the antecedent) with a simple proposition (the consequence), respectively. Intuitively, ' $p \rightarrow q$ ' means that in all possible states, if $p$ is true, then $q$ is true; ' $p \times q \rightarrow r$ ' means that if $p$ is true in the current state (e.g., 'I am inside and the door is closed') and $q$ is true in a committed 
state ('I want to be outside'), then $r$ is true in a committed state ('I have to open the door'). ${ }^{1}$

An important question for a dialogue generation model is why information flows in the first place. In other words, what is a participant's basic motivation to perform a communicative action? Psychological oriented theories about motivation, such as Maslow's hierarchy of needs (Maslow 1970), are based on assumptions of internal representations and processes such as beliefs, goal setting, expectancies and desires. We will avoid motivational concepts such as hunger, fear and sexuality, however, and borrow the concept 'homeostasis' from system theory, i.e., the process by which a system maintains a balanced state. We will assume that dialogue behaviour can be modelled as an abstract process of balancing an agent's internal belief and commitment state, bringing about that the agent believes everything the agent is committed to. Below we will distinguish various types of belief and commitment, but the basic motivation for action always comes from the imbalance between the two types of states. As the needs in Maslow's pyramid, commitments may have different priorities which follow from both the order and the type of commitments; these priorities determine the basic structure of the conversation.

\section{Mental Constructs and a Balanced State}

We will assume that the agent's cognitive state consists of a number of mental constructs and that each construct contains particular pieces of information with respect to the domain of discourse. Precisely which constructs have to be included depends on the phenomenon one wants to explain or, in a generation framework, on the rules that are needed to generate particular dialogue contributions. We motivated the use of two basic types of mental constructs in the previous section-beliefs and commitments-and assumed that the basic motivation for action is the imbalance between the belief and the commitment state of the participants. In other words, the imbalance will be considered as the driving force behind the dialogue generation process. We will now explain which mental constructs are included in our generation framework and how the generation of communicative acts can be embedded in the homeostatic process based on these mental constructs.

\footnotetext{
1 The commitment inference can be formalized in terms of possible-world semantics as follows. Let $M=$ $(W, \pi, R)$ be a model that consists of a set $W$ of states with typical elements $u, v$ and $w$, a valuation function $\pi$ which assigns a truth value $\pi_{w}(p)$ to every proposition letter $p$ in each state $w$, and $R$ is a reflexive, transitive and anti-symmetric accessibility relation. The semantics of the arrow in ' $p \times q \rightarrow r$ ' is defined as follows. Suppose $u, w \in W$ then:
}

$$
\begin{aligned}
& M, u, w \models p \times q \rightarrow r \Leftrightarrow \text { if } \pi_{u}(p)=\text { true and } \pi_{w}(q)=\text { true } \\
& \text { then on every } R \text {-path from } u \text { to } w: \exists v \text { with } \pi_{v}(r)=\text { true }
\end{aligned}
$$

In short: $r$ is instrumentally necessary for $q$ if $p$. In the pragmatic model, the expression ' $p \times q \rightarrow r$ ' is used as follows: $B_{x} p \wedge C_{x} q \Rightarrow A d d\left(C_{x} r\right)$, meaning that if $x$ believes that $p$ is true and $x$ is committed to $q$, then $x$ is also committed to $r$. 


\subsection{The Agent's Mental Constructs}

An agent $x$ 's cognitive state consists of various types of beliefs and commitments:

\section{Beliefs}

- Private belief of an agent $x$ about the domain of discourse $\left(B_{x}\right)$

- Mutual beliefs about the domain $(M B)$

- Mutual beliefs about the commitments of the partner $y\left(M B C_{y}\right)$

- Information of which the partner $y$ is ignorant $\left(I_{y}\right)$. In particular, we discern two types: ignorance with respect to the beliefs of the partner $y\left(I B_{y}\right)$ and with respect to the commitments of $x\left(I C_{y}\right)$

where mutual beliefs about the domain are considered as a subset of private beliefs.

\section{Commitments}

- Private commitments of $x$ with respect to a particular state of the domain of discourse $\left(C_{x}\right)$

- Social commitments of $x\left(S_{x}\right)$. In particular, we discern two types: with respect to the beliefs of the partner $y\left(S_{x} B_{y}\right)$ and with respect to $y$ 's commitments $\left(S_{x} C_{y}\right)$

Social commitments with respect to beliefs of the other are used to indicate that the partner has asked a question with respect to a particular piece of information about the discourse domain; social commitments with respect to the commitments of the other are used to express that the other has asked a question about his own commitments. In the latter case we may think of questions such as 'Should I take an umbrella to go to the supermarket' or 'Will I have pain when I go to the dentist?'. Intuitively, commitments fulfill two roles in this paper: First, they indicate a particular desire, i.e., a particular goal state the agent wants to be in. Second, sub-commitments indicate a particular unavoidable state, i.e., a state the agent must be in before the goal state can be achieved. Whether the intermediary state is desired or not, is of no importance in this paper.

\subsection{Achieving a Balanced Situation}

To avoid unnecessary complexity, we will make two important simplifications. First, during the dialogue, the participants have no access to a domain of discourse, i.e., they are unable to observe or manipulate particular aspects of the domain. In other words, information only flows between the two dialogue partners like, for instance, in a telephone dialogue. A second simplification is that the participants only hold positive information about the domain of discourse, i.e., negation is excluded. This implies that the two participants will never hold conflicting beliefs or commitments, and, since alleged inconsistencies will never arise, the agents will never argue about a specific statement. Information may be incorrect with respect to a particular instance of the domain of discourse, but the incorrectness will never be discovered, since the agents have no access to the domain.

A balanced situation can be achieved by updating the mental constructs of the agents. Let us therefore first define a balanced state of an agent. 
An agent $x$ has a balanced state iff

1. $x$ has no commitments or

2. (a) all private commitments $x$ are privately believed by $x$ and

(b) all social commitments of $x$ about $y$ 's beliefs are mutually believed and

(c) all social commitments of $x$ about $y$ 's commitments are mutually believed to be $y$ 's commitments

In order to achieve a balanced situation, agents may either modify their belief state or their commitment state. In line with the communication model of Sect. 3, private beliefs can in principle be modified in three ways: (a) by belief inference to make implicit beliefs explicit; (b) by an appropriate communicative act from the dialogue partner; and (c) by direct perception of the domain of discourse. In the second case, agents 'take over' the belief of the dialogue partner. Below, the third case will not be considered, since we assumed that the agents have no access to the domain of discourse. In the presented dialogue game, explicit beliefs can thus only be modified in two ways: via a reasoning mechanism for the belief states of the agents and by communication with the partner. Since we deal with an ideal communication channel and since we did not include negation, all manifested beliefs will be included in the agents' mutual beliefs about the domain. In practice, two versions of mutual belief exist, $x$ 's version and $y$ 's version, but since we will assume that both versions contain the same information, we will speak of one version only.

An initial commitment state can in principle be adjusted in four ways: (a) by commitment inference to make implicit private commitments explicit; (b) if the agent receives particular information with respect to its private commitment state; (c) by a question of the partner (inducing a social commitment); and (d) if the agent concludes that he is unable to change the belief state in such a way that the situation can be balanced (c.f. Cohen and Levesque 1990). In the fourth case the commitment will be cancelled.

\section{The Dialogue Game}

The dialogue game is divided into two parts (for a similar approach, see Piwek 1998 or Amgoud et al. 2000): (a) the game-board that contains information about the cognitive states and the communicative acts; and (b) the dialogue rules that control the behaviour of the participants (generation rules) and that prescribe how the game-board changes (update rules). The game-board represents the participants' cognitive state and typically changes because of the participants' communicative actions.

'Moves' or information flows between the two participants are composed of two elements: (a) plain information about the domain of discourse (the semantic content); and (b) information about the way the different mental constructs should be updated (the communicative function). Every move is completely determined by the cognitive state of the participant who has the turn to act and by the rules for co-operative behaviour that will be presented below. Since the cognitive states are updated after every move, the next move is not only determined by the previous one, as would be the case in a dialogue grammar, but also by the context of the move. Each play is a sequence—not necessarily finite — of linearly or hierarchically alternating moves. 
In the dialogue game and in line with the Gricean maxims (Grice 1975), agents do not put forward information they do not believe or information they mutually believe. The distinction between private and mutual beliefs enables us to give concrete form to the maxim of quantity. If relevant, private beliefs can always be manifested, unless they are part of the mutual beliefs. Mutual beliefs give us a criterion to leave out particular information in the dialogue contribution (otherwise we would manifest information that the other already believes). In the dialogue game, agents also do not ask information they believe or they believe the other is ignorant about.

\subsection{The Agents' Cognitive State}

All constructs of an agent's cognitive state are modelled as sets of information items, except for the private and social commitments which are both modelled as a list. The two types of social commitments together form one list.

We adopt the following shorthand notations: we write $B_{x} p$ as a shorthand for $p \in B_{x}$ and write $\neg B_{x} p$ for $p \notin B_{x}$, and similarly for the other mental constructs.

Note that in writing expressions like $B_{x} p$ (' $x$ believes that $p$ '), $B_{x}$ is not to be confused with a modal operator with corresponding semantics in terms of accessibility relations and possible worlds. Our focus is here on dialogue generation, so $B_{x}$ is instead modelled as a dynamic state with an operational semantics given by the rules of the dialogue. In particular, the rules make use of the fact that particular information $p$ is present $\left(B_{x} p\right)$, is absent $\left(\neg B_{x} p\right)$, is added $\left(\operatorname{Add}\left(B_{x} p\right)\right)$ or is deleted $\left(\operatorname{Del}\left(B_{x} p\right)\right)$.

We use the notation $S_{x} \underline{p}$ or $C_{x} \underline{p}$ to denote that $p$ is the commitment under discussion. A commitment list may be empty, indicated by $S_{x} \varnothing$ and $C_{x} \varnothing$.

\subsubsection{Inference Rules}

We assume that the agents can reason about their beliefs and commitments by the following inference rules:

$$
\begin{array}{ll}
\text { I1 } & B_{x} p \wedge B_{x}(p \rightarrow q) \Rightarrow \operatorname{Add}\left(B_{x} q\right) \\
\text { I2(a) } \quad B_{x} p \wedge C_{x} r \wedge M B(p \times r \rightarrow q) \Rightarrow \operatorname{Add}\left(C_{x} q\right) \\
\text { I2(b) } \quad M B p \wedge M B C_{x} r \wedge M B(p \times r \rightarrow q) \Rightarrow \operatorname{Add}\left(M B C_{x} q\right)
\end{array}
$$

So, if the belief base of agent $x$ contains the information that $p$ and that $p \rightarrow q$ then according to rule I1 it is updated with the information that $q$. Additionally, if $x$ believes $p$, is committed to $r$ and $p \times r \rightarrow q$ is part of the mutual beliefs then according to rule I2(a) the private commitment base is updated with $q$. Rule I2(b) is similar. To keep things simple, we will assume that all commitment inferences are part of the mutual beliefs.

The belief states are monotonic, i.e., everything that can be inferred from previous states, can also be inferred from new belief states. Information about commitments can be retracted after particular communicative acts, for instance, if an agent receives the answer 'Don't know' to his question 'whether $p$ ' then the private commitment $p$ is dropped. We are not concerned with the full details of the update mechanism, but 
assume that the cognitive states are updated in line with the principles I1 and I2 and the update rules presented below.

In the dialogue model, we assume that after each round an agent's beliefs and commitments are closed by (successive) application of the inference rules I1 and I2.

Finally, in the dialogue model, we will use a function link that gives us the set of all antecedents that are connected to a particular consequence of a belief inference. More precisely, link is defined in the following way:

$$
\operatorname{link}(x, q) \equiv\left\{p \mid B_{x}(p \rightarrow q)\right\}
$$

For instance, if $x$ believes that ' $p \rightarrow q$ ' and believes that ' $r \rightarrow q$ ', then $\operatorname{link}(x, q)=$ $\{p, r\}$. If there is no compound proposition with $q$ as its consequence in belief state $x$, the set is empty $(\varnothing)$.

\subsection{Communicative Acts}

Agents manifest their beliefs and commitments by means of communicative acts or moves, such as statements and questions. The content of a move consists of a formula: $C p$ (' $p$ is a commitment'), $B p$ (' $p$ is believed') or $C p \hookleftarrow B q$ (' $p$ is a commitment since $q$ is believed'); the communicative function is tagged by one of the following markers ('?', '!', ‘*' and '\$'):

- Questions: $[B p]^{?}$ and $[C p]^{\text {? }}$

- Statements: $[B p]^{!},[C p]^{!}$and $[C p \hookleftarrow B q]^{!}$

- Ignorance: $[B p]^{*}$ and $[C p]^{*}$

- Closure of the dialogue: []$^{\infty}$

We use the notation ' $x: m$ ' to denote that agent $x$ is the performer of the move $m$.

\subsection{Generation Rules}

The general dialogue mechanism is as follows.

1. An agent's first priority is to resolve any imbalance with respect to its social commitments. For instance, if it receives the question 'whether $p$ ' and $p$ is part of private beliefs then it responds that $p$ holds.

2. If there are no such imbalances, then the second priority is to ask questions that allow the derivation of sub-commitments of its commitments. For instance, if the agent believes $p \times r \rightarrow q$, is committed to $r$ and not (yet) to the sub-commitment $q$ then it may ask whether $p$ holds.

3. Otherwise the agent aims at resolving any imbalance between its private beliefs and commitments. For instance, if the agent is committed to $p$ but does not (yet) know that $p$, then it asks whether $p$, holds.

4. If there are no imbalances, the agent closes the dialogue.

With respect to the generation of sub-commitments in step 2. we discern two options: we can either ask for the belief $(p)$ or for the sub-commitment $(q)$. For 
instance, if we are committed to prepare dinner we prefer asking for the belief 'Are our guests vegetarian?' to asking for the (many) sub-commitments it would yield ('Do I need to buy eggs?', 'Do I need to go to the greengrocer's?', 'Do I need to soak beans?' and so on). Conversely, if we are committed to drive home we prefer to ask for the sub-commitment 'Do I need to take the road E12?' to asking for one of the (many) beliefs from which this particular commitment could be derived ('Is there a traffic jam on the E27?', 'Is the E38 still road-blocked?', 'Is the Prins Claus Bridge closed?' and so on). Hence, in the dialogue model we adopt the following (simple) strategy: given the rule $p \times r \rightarrow q$ an agent asks for the belief $p$ (rather than for the sub-commitment $q$ ) if there is at least an additional rule $p \times r \rightarrow q^{\prime}$. The agent asks for the the sub-commitment $q$ (rather than for the belief $p$ ) if there is at least an additional rule $p^{\prime} \times r \rightarrow q$. If neither or both of these conditions hold then the agent chooses randomly. ${ }^{2}$

Speech acts (or moves) are fully determined by the cognitive state of the participant who performs the move and by the rules that are applicable to this state. The double arrow ' $\Rightarrow$ ' links the preconditions of the move to the move itself. The left side of the arrow is of type proposition and represents the preconditions in terms of the cognitive state of an agent; the right side is of type action and represents the generated move.

\subsubsection{Questions}

Since the agents have no access to the domain of discourse, the initial move can only be a question. There are two types of questions. The first is a question whether some proposition is believed to hold:

$$
\text { G0. } C_{x} p \wedge \neg B_{x} p \wedge S_{x} \emptyset \Rightarrow x:[B p]^{?}
$$

The first two preconditions of G0 indicate that the agent's state is out of balance with respect to the commitment $p$, the third condition indicates that there are no social commitments to be handled first. We assume that the imbalance with respect to $p$ is to be resolved by communication (and not for instance by means of actions and/or observations in the world).

The second reason for asking whether some proposition is believed to hold is that it would yield the generation of a new private commitment (according to inference rule $\mathrm{I} 2$ ).

We first introduce the following short-hand notation $\Phi$ :

$$
\Phi(x, p, r, q) \equiv M B(p \times r \rightarrow q) \wedge C_{x} r \wedge \neg C_{x} q
$$

which expresses that the agent believes $p \times r \rightarrow q$, has $r$ as its private commitment and does not have $q$ as a private commitment (yet). The dialogue rules are then as follows:

\footnotetext{
2 For the purposes of this paper, we consider this simple preference ordering. More involved orderings could for instance take the number and/or priorities of beliefs and sub-commitments into account.
} 


$$
\begin{aligned}
& \text { G1. } \Phi(x, p, r, q) \wedge \neg I B_{y} p \wedge S_{x} \varnothing \Rightarrow x:[B p]^{?} \\
& \text { G2. } \Phi(x, p, r, q) \wedge \neg I C_{y} q \wedge S_{x} \varnothing \Rightarrow x:[C q]^{?}
\end{aligned}
$$

The first precondition indicates that a new sub-commitment $q$ of $r$ could be developed from the belief that $p$. The question whether this proposition $p$ holds can be asked if the agent does not believe that the other is ignorant. Similar to rule G0 it is also required that there are no social commitments to be handled first.

In order to decide between the application of rule $\mathrm{G} 1$ and $\mathrm{G} 2$ we define the following strategy. Given $x$ and $r$,

- prefer G1 if there exist at least two distinct instances of $q$

- prefer G2 if there exist at least two distinct instances of $p$

- if both or none are preferred then choose randomly

So for instance, if $\Phi(x, p, r, q)$ and $\Phi\left(x, p, r, q^{\prime}\right)$ hold then ask for the belief $p$, if $\Phi(x, p, r, q)$ and $\Phi\left(x, p^{\prime}, r, q\right)$ hold then ask for the commitment $q$, and if all of these conditions hold then either ask for the belief $p$ or for the commitment $q$.

\subsubsection{Responses to Questions}

After the initiator has asked a question, his question becomes manifest as a social commitment of the follower. There are three possibilities for the next move:

a. The follower knows the answer and thus gives the answer. If applicable she even makes a more cooperative move by including a relevant explanation.

b. The follower does not know the answer directly, but concludes that there may be a way to find the answer and asks a counter-question.

c. The follower is ignorant and does not have a solution. She manifests her ignorance.

With respect to case a. an explanation is relevant if it allows for the generation of additional sub-commitments. For instance, given the question 'Do I need to come home?', adding an explanation (e.g., 'because your dog is sick') to the answer 'Yes' is relevant if it would allow the other to infer one or more additional sub-commitments (such as 'I need to hurry.').

Generation rule $\mathrm{G} 3$ expresses that if $x$ has the social commitment with respect to the belief $q$ of $y$ and $q$ is believed by $x$, then $x$ will answer that $q$ is believed to hold:

$$
\text { G3. } S_{x} B_{y} \underline{q} \wedge B_{x} q \Rightarrow x:[B q]^{!}
$$

Additionally, if $x$ has the social commitment with respect to the commitment $q$ of $y$ and the commitment $q$ can be inferred from the belief that $r$, then if $r$ is relevant, then $x$ will answer that $q$ is a commitment because $r$ is believed (G4), otherwise $x$ will just answer that $q$ is a commitment (G5).

$$
\begin{aligned}
& \text { G4. } S_{x} C_{y} \underline{q} \wedge \Psi(x, p, r, q) \wedge B_{x} p \wedge \operatorname{relevant}(x, p, r, q) \Rightarrow x:[C q \hookleftarrow B p]^{!} \\
& \text {G5. } S_{x} C_{y} \underline{q} \wedge \Psi(x, p, r, q) \wedge B_{x} p \wedge \neg \operatorname{relevant}(x, p, r, q) \Rightarrow x:[C q]^{!}
\end{aligned}
$$


where we use the short-hand notation:

$$
\Psi(x, p, r, q) \equiv M B(p \times r \rightarrow q) \wedge M B C_{y} r
$$

to express that the agent believes $p \times r \rightarrow q$ and it is mutually believed that $r$ is a private commitment of the other.

Additionally, the notion of relevance is formalised as follows: ${ }^{3}$

$$
\operatorname{relevant}(x, p, r, q) \equiv \exists q^{\prime} \neq q \wedge \Psi\left(x, p, r, q^{\prime}\right) \wedge \neg M B C_{y} q^{\prime}
$$

which expresses that $p$ (e.g., 'dog is sick') is relevant if it would allow the generation of an additional commitment $q^{\prime}$ ('need to hurry') in addition to $q$ ('need to come home').

Additionally, if $x$ does not know the answer, $x$ may ask a counter-question. The counter-question can only be asked if the agent finds the antecedent of a linked proposition, and if he or she does not believe that the other is ignorant with respect to the linked proposition (G6 and G7):

$$
\begin{aligned}
& \text { G6. } S_{x} B_{y} \underline{q} \wedge \neg B_{x} q \wedge p \in \operatorname{link}(x, q) \wedge \neg I B_{y} p \Rightarrow x:[B p]^{?} \\
& \text { G7. } S_{x} C_{y} \underline{q} \wedge \Psi(x, s, r, q) \wedge \neg B_{x} s \wedge p \in \operatorname{link}(x, s) \wedge \neg I B_{y} p \Rightarrow x:[B p]^{?}
\end{aligned}
$$

For reasons of legibility we have omitted from rule $\mathrm{G} 7$ the preconditions implying that neither rule G4 nor G5 is applicable.

If $x$ does not know the answer and cannot ask a counter-question, $x$ will manifest his or her ignorance (G8 and G9).

$$
\begin{aligned}
& \text { G8. } S_{x} B_{y} \underline{q} \wedge \neg B_{x} q \wedge \neg\left(\exists p: p \in \operatorname{link}(x, q) \wedge \neg I B_{y} p\right) \Rightarrow x:[B q]^{*} \\
& \text { G9. } S_{x} C_{y} \underline{q} \wedge \text { not_applicable }(G 7) \Rightarrow x:[C q]^{*}
\end{aligned}
$$

where for reasons of legibility we use the notation not_applicable $(G 7)$ to express that the precondition of $\mathrm{G} 7$ does not hold.

Finally, in G10 a closing act is generated if the social commitment list is empty and if the situation is in balance:

$$
\text { G10. } S_{x} \varnothing \wedge \neg \exists q:\left(C_{x} q \wedge \neg B_{x} q\right) \Rightarrow x:[]^{2}
$$

To avoid an infinite sequence of closing acts, a meta-rule has been defined to close the dialogue:

Closing $(C L)$ : Both dialogue partners stop generating communicative acts iff two successive closing acts are performed (i.e., the sequence $x:[]^{\boldsymbol{\alpha}}$ and $y:[]^{\boldsymbol{\alpha}}$ ).

\footnotetext{
3 For the purposes of this paper we consider a simple notion of relevance.
} 


\subsection{The Update of Cognitive States}

The update function yields a new cognitive state depending on the old state and the move just performed. To represent the consequences of a particular move, we use the notation ' $\Rightarrow$ '. The left side is of type action and represents the performed move; the right side represents how the cognitive states should be updated. The relevant attitudes are preceded by $\mathrm{Del}$ or Add depending on whether information is to be added or removed. So, for instance, $\operatorname{Del}\left(C_{x} q\right)$ means that $q$ should be deleted from the private commitments list.

In update rule $\mathrm{U} 1$, it is expressed that if $x$ utters a statement that $q$ holds then $q$ is added to the mutual beliefs.

$$
\text { U1. } x:[B q]^{!} \Rightarrow \operatorname{Add}(M B q)
$$

Update rule $\mathrm{U} 2$ indicates that if $x$ utters that $q$ is a commitment then this becomes a mutual belief.

$$
\text { U2. } x:[C q]^{!} \Rightarrow \operatorname{Add}\left(M B C_{y} q\right)
$$

Update rule $\mathrm{U} 3$ is similar to $\mathrm{U} 2$. In addition the explanation $p$ is added to the mutual beliefs.

$$
\text { U3. } x:[C q \hookleftarrow B p]^{!} \Rightarrow \operatorname{Add}(M B p) \wedge A d d\left(M B C_{y} q\right)
$$

Rules U4 expresses that if $x$ utters a question whether $q$ holds, then $y$ obtains the social commitment with respect to the belief $q$ of $x$.

$$
\text { U4. } x:[B q]^{?} \Rightarrow \operatorname{Add}\left(S_{y} B_{x} q\right)
$$

If $x$ utters a question whether $q$ is a commitment, then $y$ obtains the social commitment with respect to the commitment $q$ of $x$ (U5):

$$
\text { U5. } x:[C q]^{?} \Rightarrow \operatorname{Add}\left(S_{y} C_{x} q\right)
$$

Rule U6 and U7 express that if $x$ indicates that he or she has no information about (the belief or commitment) $q, q$ will be added to the beliefs of $y$ about the ignorance of $x$ and removed from the social commitments and, if present, from the private commitments of $y$ :

$$
\begin{aligned}
& \text { U6. } x:[B q]^{*} \Rightarrow \operatorname{Add}\left(I B_{x} q\right) \wedge \operatorname{Del}\left(S_{x} B_{y} q\right) \wedge \operatorname{Del}\left(C_{y} q\right) \\
& \text { U7. } x:[C q]^{*} \Rightarrow \operatorname{Add}\left(I C_{x} q\right) \wedge \operatorname{Del}\left(S_{x} C_{y} q\right)
\end{aligned}
$$

The last rule, U8, expresses that cognitive states do not change after a closing act:

$$
\text { U8. } x:[]^{*} \Rightarrow \otimes
$$


Finally, we assume the following two belief state maintenance rules:

$$
\begin{aligned}
& M 1 . A d d(M B p) \Rightarrow \operatorname{Add}\left(B_{x} p\right) \wedge \operatorname{Add}\left(B_{y} p\right) \wedge \operatorname{Del}\left(I B_{y} p\right) \wedge \operatorname{Del}\left(I B_{x} p\right) \\
& M 2 . A d d\left(M B C_{x} p\right) \Rightarrow \operatorname{Add}\left(C_{x} p\right) \wedge \operatorname{Del}\left(I C_{x} p\right)
\end{aligned}
$$

Rule M1 indicates that if $p$ becomes mutual knowledge, it is added to the private beliefs and if present removed from the beliefs about the other agent's ignorance. According to rule M2, if $p$ is added to the mutual beliefs about the commitments of agent $x$, it is added to the commitments of $x$ as well and deleted from the ignorance of $y$ about $x$ 's commitments.

\section{A Dialogue Example}

We turn now to an example where John and Mary play the co-operative dialogue game based on the previously introduced mental constructs, and the inference, generation, update and maintenance rules. First, we present an abstract version of the example, and after that, we will translate the example into a 'natural' language dialogue.

In Fig. 2, we have depicted the dialogue transition table, i.e., the cognitive states of Mary and John, the communicative acts (MOVE) and, in addition, a reference to the applied update and generation rules. Empty states are indicated by ' $\varnothing$ '. In the example, we have left out Mary's commitment state $\left(C_{M}\right)$, Mary's belief state about John's ignorance $\left(I_{J}\right)$, the mutual beliefs about Mary's commitments $\left(M B C_{M}\right)$ and John's social commitments with respect to the commitments of Mary $\left(S_{J} C_{M}\right)$, since in this particular situation these states remain empty during the course of the dialogue. In the initial situation, the beliefs are as follows (because of space limitations we have not depicted them in the table). John and Mary mutually believe that:

1. if one is late and wants to go to the supermarket then one needs to hurry (' $l \times s \rightarrow$ $\left.h^{\prime}\right)$

2. if one is late and wants to go to the supermarket then one needs to go by bike (' $l \times s \rightarrow b$ ')

3. if there is a road-block and one wants to go by bike then one needs to take the detour (' $r b \times b \rightarrow d$ ')

4. if the bridge is closed and one wants to go by bike then one needs to take the detour (' $b c \times b \rightarrow d$ ')

5. if it is raining and one wants to go by bike then one needs to take a jacket (' $r \times b \rightarrow$ $\left.j^{\prime}\right)$

6. if it is raining and one wants to go by bike then one needs to take an umbrella (' $r \times b \rightarrow u$ ')

7. if it is cold and one wants to go by bike then one needs to take a jacket (' $c \times b \rightarrow j$ ')

8. if all umbrella's are currently in use and one wants to take an umbrella one needs to fetch one from the attic (' $u u \times u \rightarrow a$ ')

9. if all umbrella's are broken and one wants to take an umbrella one needs to fetch one from the attic (' $u b \times u \rightarrow a$ ') 


\begin{tabular}{|c|c|c|c|c|c|c|c|c|c|c|}
\hline & Mary & & & & & & John & & & \\
\hline Nr. & $B$ & $S B_{\mathrm{J}}$ & $S C_{J}$ & $M B C_{J}$ & $M B$ & MOVE & $C$ & $B$ & $S B_{M}$ & $I C_{M}$ \\
\hline $\begin{array}{l}\text { G1 } \\
1 .\end{array}$ & $l, r, w \rightarrow u u$ & $\theta$ & $\emptyset$ & $s$ & $\emptyset$ & $J:[B l] ?$ & $s$ & $w$ & $\theta$ & \\
\hline $\begin{array}{l}\text { U4 } \\
\text { G3 } \\
2 .\end{array}$ & & $\underline{l}$ & & & & $M:[B l] !$ & & & & \\
\hline $\begin{array}{l}\text { U1 } \\
\text { M1 } \\
\text { I2 } \\
\text { G2 } \\
3 .\end{array}$ & & & & $h, b$ & $l$ & $J:[C d] ?$ & $h, b$ & $l$ & & \\
\hline $\begin{array}{l}\text { U5 } \\
\text { G9 } \\
4 .\end{array}$ & & & $\underline{d}$ & & & $M:[C d]^{*}$ & & & & \\
\hline $\begin{array}{l}\mathrm{U} 7 \\
\text { G2 } \\
5 .\end{array}$ & & & $\emptyset$ & & & $J:[C j] ?$ & & & & $d$ \\
\hline $\begin{array}{l}\text { U5 } \\
\text { G4 } \\
6 .\end{array}$ & & & $\underline{j}$ & & & $M:[C j \hookleftarrow B r] !$ & & & & \\
\hline $\begin{array}{l}\mathrm{U} 3 \\
\mathrm{M} 1 \\
\mathrm{I} 2 \\
\mathrm{M} 2 \\
\mathrm{G} 2 \\
7 .\end{array}$ & & & & $\begin{array}{l}j \\
u\end{array}$ & $r$ & $J:[C a] ?$ & $\begin{array}{l}u \\
j\end{array}$ & $r$ & & \\
\hline $\begin{array}{l}\text { U5 } \\
\text { G7 } \\
8 .\end{array}$ & & & $\underline{a}$ & & & $M:[B w] ?$ & & & & \\
\hline $\begin{array}{l}\text { U4 } \\
\text { G3 } \\
9 .\end{array}$ & & & & & & $J:[B w] !$ & & & $\underline{w}$ & \\
\hline $\begin{array}{l}\text { U1 } \\
\text { M1 } \\
\text { I1 } \\
\text { G5 } \\
10 .\end{array}$ & $\begin{array}{l}w \\
u u\end{array}$ & & & & $w$ & $M:[\mathrm{Ca}] !$ & & & & \\
\hline $\begin{array}{l}\text { U2 } \\
\text { M2 } \\
\text { G10 } \\
11 .\end{array}$ & & & & $a$ & & $J:[]^{\infty}$ & $a$ & & & \\
\hline $\begin{array}{l}\text { U8 } \\
\text { G10 } \\
12 .\end{array}$ & & & & & & $M:[]^{\boldsymbol{*}}$ & & & & \\
\hline $\begin{array}{l}\mathrm{U} 8 \\
\mathrm{CL}\end{array}$ & & & & & & & & & & \\
\hline
\end{tabular}

Fig. 2 Dialogue between John and Mary about John's commitment to go to the supermarket. On the basis of Mary's responses various sub-commitments (e.g., going by bike, taking an umbrella) are generated

Mary believes that John is late $(l)$, that it is raining $(r)$ and that if William is at school (and thus has taken his umbrella) then all umbrella's are currently in use $(w \rightarrow u u)$. Mary has no commitments. John believes that William is at school $(w)$. It is mutually believed that John is committed to go to the supermarket. John is the initiator and starts with the initial question whether $l$ (move 1). Mary is able to answer this question directly (move 2). From the answer it is concluded that John has to hurry $(h)$ and has to go by bike $(b)$. Based on his new commitment to go by bike John asks whether he has to take the detour $(d)$ (move 3). Mary informs that she has no information about this (move 4). John asks whether he needs to take a jacket ( $j$ ) (move 5). Mary answers that he indeed needs one because it is raining (move 6), from which John also infers that he should take an umbrella $(u)$. On the basis of this new commitment, John asks whether he needs to fetch one from the attic (a) (move 7). Mary is unable to answer this question directly, but may find an answer if she has the information that 
William is at school $(w)$. So, she asks for this information (move 8). John answers that this is indeed the case (move 9), from which Mary infers that John should fetch an umbrella from the attic. She is now able to answer his question (move 10). Finally, since the remaining imbalance between beliefs and commitments cannot be solved by communication (but instead have to be resolved by other means such as actions and observations), the dialogue is closed (move 11 and move 12).

Below the corresponding dialogue is presented in 'natural language':

0. John: I want to go to the supermarket. (not manifested in the transition table)

1. John: Am I late?

2. Mary: Yes.

3. John: Do I need to take the detour then?

4. Mary: That I don't know.

5. John: And do I need to take my jacket?

6. Mary: Yes, because it is raining.

7. John: Do I need to fetch an umbrella from the attic then?

8. Mary: Is William at school?

9. John: Yes.

10. Mary: Well in that case you need to fetch one from the attic.

11. John: OK, thank you.

12. Mary: OK, no thanks.

\section{Discussion and Future Research}

In this paper we presented a framework for the generation of coherent elementary conversational sequences at the speech act level. We were able to show by means of an explicit presentation of the transition tables that the structure and the coherence of conversational units are the result of an interplay between the dialogue and update rules and the initial cognitive states of the dialogue partners. Fig. 2 carefully shows the pre-and post-conditions of every speech act and the change of the mental constructs during the dialogue as a basis for utterance production. Clearly, the dialogue is still unnatural and lacks many of the ingredients that we usually observe when we study the properties of natural language dialogue. Taking a more profound look, however, we notice the elementary structural phenomena discussed at the beginning of this paper. In Fig. 2 we can observe the linear organisation of adjacency pairs, such as questionresponse (moves 1-2, and moves 3-4) and the closing of the dialogue (moves 11-12). We also observe the hierarchical organisation of insertion sequences, such as moves 8 and 9 between the question in 7 and its reply in 10. Depending on the initial states, the dialogue rules generate an arbitrary number of levels of sub-sequences and the final reply may be originated many turns away from the initial question (see also Beun 2001). The dialogue coherence, although admittedly oversimplified, comes from both the background knowledge of the dialogue partners and the way the commitments states are processed.

In our approach we extended the work presented in (Beun 2001) in several ways in order to generate richer dialogue structures. In contrast to (Beun 2001), we did not 
take a rigid non-planning approach, but included a simple possibility to reason about commitments. In (Beun 2001) a planning approach was rejected because of complexity problems and the presented communication model incorporated an extreme sensitivity to the local circumstances of the conversation. In this paper we still prefer a local solution, but admit that talking and reasoning about commitments (or goals) is a substantial aspect of conversation and therefore has to be included to generate more interesting speech act sequences. We therefore included a possibility to reason about the commitments of the participants in a very simple manner.

Another extension of the model described in (Beun 2001) is the inclusion of the participants' mutual belief. Implicitly, and in line with other researchers (e.g., Clark and Marshall 1981; Allwood et al. 1992; Traum 1994), we assumed that successful communication also requires some degree of alignment or common ground and that the goal of grounding of information is a vital activity in cooperative communication. Prior to a conversation, participants not only have beliefs of a particular discourse domain, they also assume that there is some agreement about these beliefs and they augment manifested beliefs to the 'agreed beliefs' during the conversation. In practice it is hard (and, since dialogue participants have no direct access to their partner, even impossible) to decide whether mutual beliefs are really common, but our main point is that dialogue participants act as if these beliefs are common. In fact, the introduction of mutual beliefs enabled us to give concrete form to the Gricean maxim of quantity, since mutual beliefs give a criterion to leave out particular information in the dialogue move.

The approach presented in this paper is still rudimentary and extensions could be developed along different lines, such as an extension of the domain and communication language, the addition of roles played by the agents and the investigation of different communicative situations. It should be noted, however, that some extensions may have far-reaching consequences for different aspects of the game. For instance, including negation in the domain language seems another inevitable step towards a generalisation of the framework, since it introduces the possibility of modelling conflicting beliefs and the generation of argumentative dialogues. Nevertheless, a negated proposition cannot simply be added to a belief state of one of the participants, since it may result in unwanted inconsistencies. A solution is the introduction of a temporary state that represents the beliefs of an agent about the beliefs of his or her partner- ' $A$ believes that $B$ believes' - so that the different types of information can be carefully separated and inconsistencies can be avoided. It is unclear, however, how these conflicts will ever be resolved in the present game without other extensions, since both participants are considered 'equal' and there is no reason why they would prefer one proposition over the other. In other words, an agent can never accept a conflicting proposition stated by the other if the model does not contain a notion of 'expertise' or 'power'.

Another line of research would be a careful analysis of what actually happens in human dialogues. In order to determine what humans do in realistic conversational circumstances and to validate the model presented in this paper, the acquisition of empirical data is a necessary step in the research process. We will, therefore, collect empirical data from various conversations and hope that the analysis of the transcripts 
will lead to a further extension and refinement of the model, such as the inclusion of richer semantic descriptions of the domain information.

Some important simplifications were made in the game with respect to the underlying communication model. Utterances were always accepted and did not have to be checked for inconsistencies or other counter-evidence. Participants could never be misinformed and could not have weak evidence for a specific fact. In natural situations, however, where people have multimodal access to various aspects of the world, information channels may be disturbed and an agent's attention may be attracted by a variety of sources, including pointing acts of the dialogue partner. The triangle model described in Sect. 3 includes some of the necessary basic ingredients to describe these phenomena, but there are a number of important questions left. For instance, when do agents decide to observe the domain rather than infer the information from their own belief state or ask a question to their partner? How does an agent's cognitive representation depend on whether information is observed or communicated and how do these representations influence the course of the dialogue? Partly, the answers depend on fundamental psychological issues, such as perceptual abilities, memory capacity and attention capabilities. In this paper, however, we abstracted from these matters, since including them dramatically increases the complexity of the model without supplying a substantial contribution to the explanation of the dialogue structure.

Acknowledgements The authors would like to thank Lidwien van de Wijngaert for the statistical analysis of the experimental data. We also would like to thank the three anonymous reviewers for their helpful comments on previous drafts of this paper.

\section{References}

Ahn, R., Beun, R., Borghuis, T., Bunt, H., \& Overveld, C. v. (1995). The DenK-architecture: A fundamental approach to user-interfaces. Artificial Intelligence Review, 8(9), 431-445.

Allwood, J., Nivre, J., \& Ahlsen, E. (1992). On the semantics and pragmatics of linguistic feedback. Journal of Semantics, 9, 1-26.

Amgoud, L., Maudet, N., \& Parsons, S. (2000). Modelling dialogues using argumentation. In Proceedings of the fourth international conference on multiAgent systems (ICMAS 2000), (pp. 31-38). Boston (MA)

Asher, N., \& Lascarides, A. (1998). Questions in dialogue. Linguistics and Philosophy, 23(2), 237-309.

Austin, J. (1962). How to do things with words. Oxford: Clarendon Press.

Bateman, J., \& Rondhuis, K. (1997). Coherence relations: Towards a general specification. Discourse Processes, 24, 3-49.

Beun, R. (2001). On the generation of coherent dialogue: A computational approach. Pragmatics and Cognition, 9(1), 37-68.

Bunt, H. (1989). Information dialogues as communicative action in relation to partner modelling and information processing. In M. Taylor, F. Néel, \& D. Bouwhuis (Eds.), The structure of multimodal dialogue (pp. 47-73). Amsterdam: Elsevier Science Publishers.

Carlson, L. (1985). Dialogue games. An approach to discourse analysis. Dordrecht: D. Reidel Publishing Company.

Clark, H., \& Marshall, C. (1981). Definite reference and mutual knowledge. In A. Joshi, B. Webber, \& I. Sag (Eds.), Elements of discourse understanding (pp. 10-63). Cambridge: Cambridge University Press.

Cohen, P., \& Levesque, H. (1990). Persistence, intention and commitment. In P. Cohen, J. Morgan, \& M. Pollack (Eds.), Intentions and Communication. (pp. 33-69). Cambridge, Mass.: MIT Press.

Gatt, A., \& van Deemter, K. (2006). Conceptual coherence in the generation of referring expressions. In Proceedings of the workshop on coherence for generation and dialogue (ESSLLI 2006) (pp. 17-24). Malaga. 
Gernsbacher, M., \& Givón, T. (1995). Coherence in spontaneous text. Amsterdam: John Benjamins Publishing Company.

Givón, T. (1995). Coherence in Text vs. Coherence in Mind. In M. Gernsbacher, \& T. Givón (Eds.), Coherence in spontaneous text (pp. 59-115). Amsterdam: John Benjamins Publishing Company.

Grice, H. (1975). Logic and conversation. In P. Cole, \& J. Morgan (Eds.), Speech acts. syntax and semantics, Vol. 11, (pp. 41-58). New York: Academic Press.

Grosz, B., \& Sidner, C. (1986). Attention, intentions, and the structure of discourse. Computational Linguistics, 12(3), 175-204.

Hamblin, C. (1971). Mathematical models of dialogue. Theoria, 37, 130-155.

Hobbs, J. (1979). Coherence and coreference. Cognitive Science, 3(1), 67-90.

Hulstijn, J. (2000). Dialogue models for inquiry and transaction. Ph.D. thesis, University of Twente.

Hulstijn, J., Dignum, F., \& Dastani, M. (2005). Coherence constraints for agent interaction. In R. v. Eijk, M.-P. Huget, \& F. Dignum (Eds.), Agent Communication, (Vol. 3396 of LNAI. pp. 134-152). SpringerVerlag.

Hutchins, E. (1989). Metaphors for interface design. In (Taylor et al., 1989), (pp. 11-28).

Kibble, R., \& Power, R. (2004). Optimizing referential coherence in text generation. Computational Linguistics, 30(4), 401-416.

Larsson, S., \& Traum, D. (2000). Information state and dialogue management in the TRINDI dialogue move Engine Toolkit. Natural Language Engineering, 6(3-4), 323-340.

Levinson, S. (1983). Pragmatics. Cambridge: Cambridge University Press.

Longacre, R. E. (1996). The grammar of discourse. New York: Plenum Press.

Maslow, A. (1970). Motivation and personality. New York: Harper and Row.

Piwek, P. (1998). Logic, information \& conversation. Ph.D. thesis, Eindhoven University of Technology.

Piwek, P. (2006). Meaning and dialogue coherence. In Proceedings of the workshop on coherence for generation and dialogue (ESSLLI 2006). Malaga, pp. 57-64.

Polanyi, L. (1988). A formal model of the structure of discourse. Journal of Pragmatics, 12, 601-638.

Power, R. (1979). The organisation of purposeful dialogues. Linguistics, 17, 107-152.

Redeker, G. (1990). Ideational and pragmatic markers of discourse structure. Journal of Pragmatics, 14, 367-381.

Rickheit, G., \& Strohner, H. (1992). Towards a cognitive theory of linguistic coherence. Theoretical Linguistics, 18(2/3), 209-237.

Sanders, T., \& Noordman, L. (2000). The role of coherence relations and their linguistic markers in text processing. Discourse Processes, 29(1), 37-60.

Sanders, T., Spooren, W., \& Noordman, L. (1992). Toward a taxonomy of coherence relations. Discourse Processes, 15(1), 1-35.

Singh, M. (1999). An ontology for commitments in multi-agent systems: toward a unification of normative concepts. Artificial Intelligence and Law, 7, 97-113.

Traum, D. R. (1994). A computational theory of grounding in natural language conversation. Ph.D. thesis, University of Rochester. Technical Report 545. 